\title{
Valproate-induced Drug Rash Eosinophilia with Systemic Symptoms Syndrome: An Unknown Hepatotoxicity
}

Tarana Gupta

\begin{abstract}
Drug rash eosinophilia with systemic symptoms (DRESS syndrome) presents as an acute febrile illness with leukocytosis, eosinophilia, lymphadenopathy, skin rash with acute hepatitis, renal failure, myositis, or systemic organ involvement. Aromatic anticonvulsants like phenytoin, carbamazepine, and phenobarbital cause drug-induced hypersensitivity or DRESS syndrome. However, sodium valproate being nonaromatic compound although known hepatotoxic drug in preexisting chronic liver disease has never been reported to cause DRESS syndrome alone. Here we report an interesting case of DRESS syndrome caused by valproate, which presented as an acute hepatitis illness with rash, renal dysfunction, and typical hematological features of DRESS syndrome within 2 months of the introduction of the drug in an epileptic patient. Patient initially showed a good response to intravenous steroids with improvement in the liver and renal dysfunction. However, later on, developed pancytopenia either due to steroid-induced sepsis or DRESS syndrome-related secondary hemophagocytosis (HPS) due to involvement of bone marrow as a rare occurrence and succumbed to illness.

Keywords: Drug rash eosinophilia with systemic symptoms syndrome, Hepatotoxicity, Sodium valproate.

Euroasian Journal of Hepato-Gastroenterology (2019): 10.5005/jp-journals-10018-1298
\end{abstract}

\section{BACKGROUND}

Drug rash with eosinophilia and systemic symptoms syndrome, also known as "drug-induced hypersensitivity syndrome" (DIHS) is most frequently associated with aromatic anticonvulsants like phenytoin, carbamazepine, phenobarbital and less with felbamate, oxcarbazepine, lamotrigine and zonisamide. ${ }^{1}$ Nonaromatic anticonvulsants like topiramate, levetiracetam, valproate are relatively safe. In the absence of any chronic liver disease, sodium valproate is first-line therapy in epilepsy. Here we present a rare case of acute hepatitis with full-blown DRESS syndrome due to sodium valproate.

\section{Case Description}

A 45-year-old female presented with a history of maculopapular rash for 15 days, followed by jaundice for 7 days and fever of 1-day duration. She was a known epileptic started on sodium valproate 1,000 mg per day in two divided doses was added for 2 months for uncontrolled seizures. On physical examination, maculopapular lesions on the chest, abdomen, back, and upper limbs were observed. She had tachycardia, with pulse rate 110/minute, vitals were stable, her blood pressure was $124 / 78 \mathrm{~mm} \mathrm{Hg}$. Icterus was present, no lymphadenopathy was identified. Investigations revealed leukocytosis with total leukocyte count $15,000 / \mathrm{mm}^{3}$ with eosinophilia, absolute eosinophil count $1,800 / \mathrm{mm}^{3}$, and normal platelet count. Her total bilirubin reached up to $16.9 \mathrm{mg} / \mathrm{dL}$ with direct fraction $9.6 \mathrm{mg} / \mathrm{dL}$, aspartate aminotransferase (AST) 1,180 $\mathrm{U} / \mathrm{L}$, alanine aminotransferase (ALT) $687 \mathrm{U} / \mathrm{L}$, alkaline phosphatase (ALP) $465 \mathrm{U} / \mathrm{L}$ and prothrombin time was 25.1 seconds with INR 2.1. Her viral markers, $A, B, C$, and $E$, including antinuclear antibody (ANA), were negative. On admission, she had mild renal dysfunction with blood urea $80 \mathrm{mg} / \mathrm{dL}$ and creatinine of $2 \mathrm{mg} / \mathrm{dL}$, which later improved with hydration. In the background of the recent introduction of sodium valproate and new-onset jaundice with skin rash, eosinophilia, negative viral markers, and ANA, a possibility
Department of Medicine, Pandit Bhagwat Dayal Sharma Post Graduate Institute of Medical Sciences, Rohtak, Haryana, India

Address reprint requests to: Tarana Gupta, Department of Medicine, Pandit Bhagwat Dayal Sharma Post Graduate Institute of Medical Sciences, Rohtak, Haryana, India, Phone: +91 1262-211307, e-mail: taranagupta@gmail.com

How to cite this article: Gupta T. Valproate-induced Drug Rash Eosinophilia with Systemic Symptoms Syndrome: An Unknown Hepatotoxicity. Euroasian J Hepato-Gastroenterol 2019;9(2):102-103.

Source of support: Nil

Conflict of interest: None

of DRESS syndrome was kept. Patient was started on intravenous antibiotics with dexamethasone. Patient initially showed improvement with a reduction in leukocytosis, eosinophilia, and total bilirubin to $11.7 \mathrm{mg} / \mathrm{dL}$. There was an improvement in skin rashes and general well-being. However, after day 8 , the clinical course again worsened, and swelling with marked tenderness of the right thigh was observed. Magnetic resonance imaging (MRI) of right thigh was suggestive of myositis. Her investigations showed the development of pancytopenia with hemoglobin $5 \mathrm{~g} / \mathrm{dL}$, total leukocyte count $1,000 / \mathrm{mm}^{3}$, platelet count $20,000 / \mathrm{mm}^{3}$, worsening jaundice with total bilirubin $16.5 \mathrm{mg} / \mathrm{dL}$, and normal liver enzymes. Her blood culture revealed Acinetobacter baumannii sensitive to colistin and resistant to carbapenems. In view of worsening sepsis with pancytopenia, antibiotics were upgraded, and steroids were stopped. Her LDH and ferritin were normal, with a corrected reticulocyte count of $0.5 \%$. Patient went into shock and despite all resuscitative measures, succumbed to her illness.

\section{Discussion}

Sodium valproate is the first-line therapy for epilepsy. It causes an elevation in aminotransferases in $0-44 \%$ of patients, with an overall incidence of $11 \% .^{2}$ Valproate most commonly causes 
microvesicular fatty infiltration of liver, which may be related to one of its metabolite 2-propyl-4-pentenoic acid. ${ }^{3}$ The hepatotoxicity of valproate usually manifests in the first 3 months of starting therapy, and close monitoring of liver function tests is needed during this period. Overall hepatic failure due to valproate toxicity is not a common occurrence. On the contrary, aromatic anticonvulsants like phenytoin, carbamazepine, and phenobarbital undergo aromatic hydroxylation with the formation of arene oxides, which are later detoxified by epoxide hydrolases producing nontoxic metabolites. In epoxide hydrolase deficiency or absence, arene toxic metabolites accumulate, resulting in direct cellular or hypersensitivity reactions like DIHS or DRESS syndrome. Although valproate is a nonaromatic anticonvulsant, drug interactions with lamotrigine can predispose to hepatotoxicity. However, valproate alone causing DRESS syndrome is a very rare occurrence. Only two cases report were found in literature search. ${ }^{4}$ The other pathophysiological factors responsible for the causation of DRESS syndrome are activation of human herpesvirus- 6 (HHV-6) and association of certain human leukocyte antigens (HLA). ${ }^{5}$ But no clear cause has been attributed to valproate till date.

Our patient typically presented with jaundice and rash around four weeks after introducing sodium valproate, suggesting a temporal association with the drug and all negative etiological workup for acute hepatitis. The maculopapular rash was followed by eosinophilia and jaundice. The peak of bilirubin followed the normalization of eosinophil count. As per European Registry of Severe Cutaneous Adverse Drug Reactions (regiSCAR) criteria, ${ }^{6}$ our patient qualified nine points with a positive history of fever, rash $>50 \%$ body surface area involvement, eosinophilia, jaundice, renal dysfunction, myositis, and negative viral and autoimmune markers and finally developing bone marrow failure. ${ }^{4}$ Patient responded well to introduction of steroids. However, our patient succumbed to sepsis with positive blood culture for the resistant strain of $A$. baumannii. It is interesting to emphasize the presence of pancytopenia pre-terminally in the index patient. The presence of pancytopenia in a setting of DRESS syndrome can be due to either severe sepsis or drug-induced or may be due to HPS resulting from DRESS syndrome itself. Although we could not perform bone marrow examination as patient was hemodynamically unstable, however, HPS cannot be ruled out in our patient.

These patients in milder forms respond well to the withdrawal of offending drug and intravenous corticosteroids (prednisone $1 \mathrm{mg} / \mathrm{kg} /$ day or equivalent). In case of worsening, IV methylprednisolone $30 \mathrm{mg} / \mathrm{kg} /$ day for 3 days can be considered. Some investigators have shown intravenous immunoglobulin (IVIG) to be useful in DIHS. ${ }^{7}$ Due to the rarity of this syndrome, a clear guideline or algorithm for the management of these patients is lacking, and therefore a case-to-case decision making should be done.

\section{Conclusion}

Sodium valproate is associated with hepatotoxicity commonly, but DRESS syndrome is a very rare occurrence. In the initial 3 months of starting valproate, close monitoring of liver function tests should be done as most hepatotoxicities are manifested during this time. In a patient presenting with rash and jaundice in the background history of sodium valproate, DRESS syndrome should also be considered, as timely recognition and prompt management can improve the outcome of these patients.

\section{Clinical Significance}

Drug toxicities should be identified early so that withdrawal of offending drug leads to improved outcome of patient.

\section{ACKnowledgments}

I wish to thank team of my resident doctors and nursing staff for giving best care to the patient.

\section{References}

1. Bohan KH, Mansuri TF, Wilson NM. Anticonvulsant hypersensitivity syndrome: implications for pharmaceutical care. Pharmacotherapy 2007;27(10):1425-1439. DOI: 10.1592/phco.27.10.1425.

2. Powell-Jackson PR, Tredger JM, Williams R. Hepatotoxicity to sodium valproate: a review. Gut 1984;25(6):673-681. DOI: 10.1136/ gut.25.6.673.

3. Lewis JH, Zimmerman HJ, Garrett CT, et al. Valproate-induced hepatic steatogenesis in rats. Hepatology 1982;2(6):870-873. DOI: 10.1002/ hep.1840020622.

4. Wu XT, Hong PW, Suolang DJ, et al. Drug-induced hypersensitivity syndrome caused by valproic acid as a monotherapy for epilepsy: first case report in Asian population. Epilepsy Behav Case Rep 2017;8:108-110. DOI: 10.1016/j.ebcr.2017.06.003.

5. Criado PR, Criado RF, Avancini JM, et al. Drug reaction with eosinophilia and systemic symptoms (DRESS)/drug-induced hypersensitivity syndrome (DIHS): a review of current concepts. An Bras Dermatol 2012;87(3):435-449. DOI: 10.1590/S0365-05962012000300013.

6. Kardaun $\mathrm{SH}$, Sekula $\mathrm{P}$, Valeyrie-Allanore L, et al. Drug reaction with eosinophilia and systemic symptoms (DRESS): an original multisystem adverse drug reaction. Results from the prospective RegiSCAR study. Br J Dermatol 2013;169(5):1071-1080. DOI: 10.1111/bjd.12501.

7. Kito Y, Ito T, Tokura Y, et al. High-dose intravenous immunoglobulin monotherapy for drug-induced hypersensitivity syndrome. Acta Derm Venereol 2012;92(1):100-101. DOI: 10.2340/00015555-1168. 